\title{
Solitary Waves, Shock Waves and Singular Solitons of the Generalized Ostrovsky-Benjamin-Bona-Mahoney Equation
}

\author{
Houria Triki ${ }^{1}$, Zlatko Jovanoski ${ }^{2}$ and Anjan Biswas ${ }^{3,4, *}$ \\ ${ }^{1}$ Radiation Physics Laboratory, Department of Physics, Faculty of Sciences, Badji Mokhtar University, P.O. Box 12, 23000 Annaba, \\ Algeria \\ ${ }^{2}$ Applied and Industrial Mathematics Research Group, School of Physical, Environmental and Mathematical Sciences, UNSW \\ Canberra, ACT 2600, Australia \\ ${ }^{3}$ Department of Mathematical Sciences, Delaware State University, Dover, DE 19901-2277, USA \\ ${ }^{4}$ Department of Mathematics, Faculty of Sciences, King Abdulaziz University, Jeddah, Saudi Arabia
}

Received: 30 May. 2013, Revised: 13 Sep. 2013, Accepted: 14 Sep. 2013

Published online: 1 Jan. 2014

Abstract: This paper obtains the solitary wave, shock wave as well as singular soliton solutions to the generalized OstrovskyBenjamin-Bona-Mahoney ( $\mathrm{gO}-\mathrm{BBM}$ ) equation. The ansatz method is applied to obtain the solutions. Several constraint conditions for the parameters are derived that establish the existence of the soliton solutions.

Keywords: solitons, shock waves, singular solitons, ocean waves

\section{Introduction}

The theory of solitons plays a dominant role in many nonlinear dynamical systems $[1,2,3,4,5]$. Of particular interest is in the field of nonlinear optical comminications systems. Although the perfect material is currently not available, optical nonlinear media with fast response times and refractive indices that depend on intensity would be ideally suited to modulate the power and phase of optical waves for all-optical devices. These all-optical devices include fibre optic sensors, routers and large-scale optical integrated circuits $[6,7]$.

For other example, they also appear in shallow water wave flow, plasma physics, nuclear physics, oceanography, theoretical physics and among others $[8,9,10,11,12,13]$.

The dynamics of solitons are governed by several nonlinear evolution equations (NLEEs) that are studied in applied mathematics. This paper addresses one such NLEE that descibes the soliton dynamics due to ocean currents. It is the generalized OstrovskyBenjamin-Bona-Mahoney (gO-BBM) equation. The Ostrovsky equation arises in the study of geophysical fluids while the Benjamin-Bona-Mahoney (BBM) equation is studied in the context of shallow water wave dynamics $[3,14,15,16,17]$. This equation models the dynamics that is much closer to realistic situations.

The aim of this paper is to look for solitons and shock wave solutions to this equation. This is accomplished by using the ansatz method.

\section{Governing Equation}

The gO-BBM equation is given by $[18,19]$

$$
\left[u_{t}+u_{x}-\alpha\left(u^{2}\right)_{x}-\beta u_{x x t}\right]_{x}=\gamma\left(u+u^{2}\right),
$$

where $\alpha, \beta$ and $\gamma$ are constants. This is a model equation for the motion of ocean currents. In this paper the $\mathrm{gO}-\mathrm{BBM}$ equation with full nonlinearity is studied, namely

$$
\left[\left(u^{l}\right)_{t}+k^{2}\left(u^{n}\right)_{x}+a\left(u^{2 n}\right)_{x}+b\left(u^{n}\right)_{x x t}\right]_{x}=c_{1} u^{n}+c_{2} u^{2 n}
$$

\footnotetext{
*Corresponding author e-mail: biswas.anjan@gmail.com
} 
where $k, a, b, c_{1}$ and $c_{2}$ are all real constants, while $l$, $n \in Z^{+}$. For the special case when $l=n=1$ the gO-BBM equation collapses to the regular O-BBM (1).

Generally (2) is not integrable by the classical method of Inverse Scattering Transform [9]. It is always useful and desirable to construct exact analytical solutions (in particular soliton solutions) for the understanding of nonlinear physical phenomena. Based on the exact solutions, we can directly and accurately analyze the properties of propagating waves in nonlinear physical systems. In view of the enormous success of the solitary wave ansatz method for solving many NLEEs with constant and variable coefficients, we use it in to find the exact solitary waves, shock waves and singular soliton solutions to (2), for any positive integers $n$ and $l$.

\section{Solitons and Shock Waves}

This section will focus on extraction the soliton solutions to the gO-BBM equation. There are three types of solitons that will be studied in this section. They are solitary waves, shock waves and singular solitons.

\subsection{Solitary Waves}

In order to solve (2), the starting hypothesis is given by $[18,19]$

$$
u(x, t)=\frac{A}{\cosh ^{p} \tau}, \quad p>0,
$$

where

$$
\tau=B(x-v t) .
$$

Here, $A$ is the amplitude of the soliton while $v$ is the speed and $B$ is the inverse width. The exponent $p$ is unknown at this point and its value will be determined during the process of deriving the solution of this equation. Substituting (3) into (2) leads to

$$
\begin{aligned}
\frac{A^{l} p^{2} l^{2} B^{2} v}{\cosh ^{p l} \tau} & -\frac{A^{l} B^{2} v p l(p l+1)}{\cosh ^{p l+2} \tau}+\frac{b A^{n} p^{4} n^{4} B^{4} v}{\cosh ^{p n} \tau} \\
& -\frac{k^{2} p^{2} n^{2} A^{n} B^{2}}{\cosh ^{p n} \tau}+\frac{k^{2} p n(p n+1) A^{n} B^{2}}{\cosh ^{p n+2} \tau} \\
& -\frac{4 a n^{2} p^{2} A^{2 n} B^{2}}{\cosh ^{2 n p} \tau}+\frac{2 a n p(2 n p+1) A^{2 n} B^{2}}{\cosh ^{2 n p+2} \tau} \\
& -\frac{b A^{n} B^{4} p n(p n+1) v\left\{p^{2} n^{2}+(p n+2)^{2}\right\}}{\cosh ^{p n+2} \tau} \\
& +\frac{b A^{n} B^{4} v p n(p n+1)(p n+2)(p n+3)}{\cosh ^{p n+4} \tau} \\
& +\frac{c_{1} A^{n}}{\cosh ^{p n} \tau}+\frac{c_{2} A^{2 n}}{\cosh ^{2 n p} \tau}=0 .
\end{aligned}
$$

From (5), equating the exponents $2 n p+2$ and $p n+4$ gives

$$
p=\frac{2}{n},
$$

which is also obtained by equating the exponents $2 n p$ and $p n+2$. Again, from (5), equating the exponent of $\cosh ^{p l} \tau$ and $\cosh ^{p n} \tau$ leads to $l=n$. With this condition in place (2) is modified to

$$
\left[\left(u^{n}\right)_{t}+k^{2}\left(u^{n}\right)_{x}+a\left(u^{2 n}\right)_{x}+b\left(u^{n}\right)_{x x t}\right]_{x}=c_{1} u^{n}+c_{2} u^{2 n}
$$

Next, equating the exponents and the coefficients of like powers of cosh function leads to

$$
\begin{aligned}
4 B^{2}\left(k^{2}-v-4 b v B^{2}\right)-c_{1} & =0, \\
6 B^{2}\left(k^{2}-v-20 b v B^{2}\right)+\left(c_{2}-16 a B^{2}\right) A^{n} & =0, \\
a A^{n}+6 b v B^{2} & =0 .
\end{aligned}
$$

Solving this system yields

$$
\begin{array}{r}
A=\left(-\frac{3 c_{1}}{2 c_{2}}\right)^{\frac{1}{n}} \\
B=\frac{1}{2} \sqrt{\frac{c_{1}\left(b c_{2}+a\right)}{b\left(k^{2} c_{2}-a c_{1}\right)}} \\
v=\frac{a\left(k^{2} c_{2}-a c_{1}\right)}{c_{2}\left(b c_{2}+a\right)} .
\end{array}
$$

Equation (11) shows that solitons will exist as long as

$$
c_{1} c_{2}<0
$$

if $n$ is an even integer. However, if $n$ is an odd integer there is no such restriction. Also, from (12) the following restriction is obtained

$$
c_{1} b\left(b c_{2}+a\right)\left(k^{2} c_{2}-a c_{1}\right)>0
$$

Thus, the solitary wave solution (valid for any positive integer $n$ ) to the $\mathrm{gO}-\mathrm{BBM}$ equation with full nonlinearity (2) is given by

$$
\begin{aligned}
& u(x, t)=\left(-\frac{3 c_{1}}{2 c_{2}}\right)^{\frac{1}{n}} \times \\
& \operatorname{sech}^{\frac{2}{n}}\left[\frac{1}{2} \sqrt{\frac{c_{1}\left(b c_{2}+a\right)}{b\left(k^{2} c_{2}-a c_{1}\right)}}\left(x-\frac{a\left(k^{2} c_{2}-a c_{1}\right)}{c_{2}\left(b c_{2}+a\right)} t\right)\right] .
\end{aligned}
$$

\subsection{Shock waves}

Let us now calculate the shock wave solution of the $\mathrm{gO}$ BBM equation with full nonlinearity (2). It is assumed that the shock wave takes the form [12]

$$
u(x, t)=A \tanh ^{p} \tau, \quad p>0,
$$

where $\tau$ is defined above and $v$ is the speed of the shock wave. The only difference is that, in this case, the parameters $A$ and $B$ are non-zero free parameters. For shock waves, $A$ is a dialation parameter (amplitude) while 
$B$ is a measure of steepness of the shock waves. Substituting (16) into (2) yields

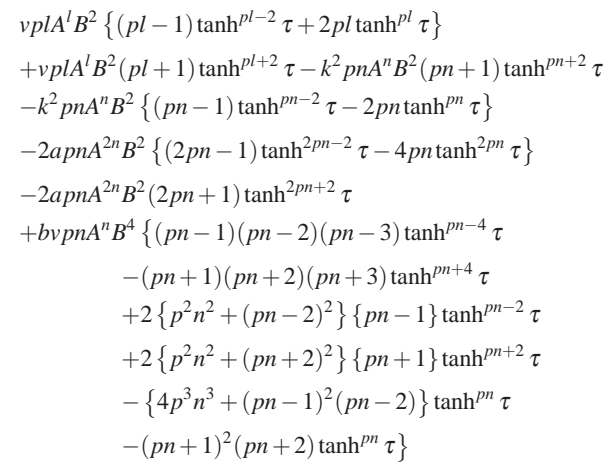

From (17), equating the exponents $2 n p+2$ and $p n+4$ gives

$$
p=\frac{2}{n}
$$

This is also arrrived at by equating the exponents of the pairs $(2 n p, p n+2)$ or $(2 n p-2, p n)$. Further, equating the exponent of $\tanh ^{p l} \tau$ and $\tanh ^{p n} \tau$ implies $l=n$. Now from (17) the linearly independent functions are $\tanh ^{p+j} \tau(j=$ $0, \pm 2, \pm 4)$ and setting their respective coefficients to zero leads to the following set of algebraic equations

$$
\begin{aligned}
k^{2}-v+8 b v B^{2} & =0, \\
6 B^{2}\left(k^{2}-v+40 b v B^{2}\right)-\left(c_{2}+32 a B^{2}\right) A^{n} & =0, \\
a A^{n}-6 b v B^{2} & =0, \\
4 B^{2}\left(3 a A^{n}+2 v-2 k^{2}-34 b v B^{2}\right)-c_{1} & =0 .
\end{aligned}
$$

Solving this system gives

$$
\begin{aligned}
v & =\frac{k^{2}}{1-8 b B^{2}} \\
A & =\left\{\frac{6 b B^{2} k^{2}}{a\left(1-8 b B^{2}\right)}\right\}^{\frac{1}{n}} \\
c_{1} & =0, \quad c_{2}=0 .
\end{aligned}
$$

It is clear that $8 b B^{2} \neq 1$ and $a \neq 0$. On account that $c_{1}=c_{2}=0$ then (2) collapses, after simplification, to the reduced $\mathrm{gO}-\mathrm{BBM}$ equation with full nonlinearity

$$
\left(u^{n}\right)_{t}+k^{2}\left(u^{n}\right)_{x}+a\left(u^{2 n}\right)_{x}+b\left(u^{n}\right)_{x x t}=0,
$$

where the integration constant is taken to be zero without any loss of generality. The shock wave solution is

$$
u(x, t)=\left\{\frac{6 b B^{2} k^{2}}{a\left(1-8 b B^{2}\right)}\right\}^{\frac{1}{n}} \tanh ^{\frac{2}{n}}\left[B\left(x-\frac{k^{2}}{1-8 b B^{2}} t\right)\right] .
$$

\subsection{Singular Solitons}

In this section, the search is for a singular soliton solution to the $\mathrm{gO}-\mathrm{BBM}$ equation with full nonlinearity (2). For this case, the ansatz is

$$
u(x, t)=A \operatorname{csch}^{p} \tau, \quad p>0 .
$$

For the case of singular solitons too, the parameters $A$ and $B$ are free non-zero parameters with $v$ being the speed of the soliton. Substituting (26) into (2) gives

$$
\begin{aligned}
& v A^{l} p^{2} l^{2} B^{2} \operatorname{csch}^{p l} \tau+p l(p l+1) v A^{l} B^{2} \operatorname{csch}^{p l+2} \tau \\
& -\left(k^{2} p^{2} n^{2} A^{n} B^{2}-b v A^{n} p^{4} n^{4} B^{4}-c_{1} A^{n}\right) \operatorname{csch}^{p n} \tau \\
& -k^{2} p n(p n+1) A^{n} B^{2} \operatorname{csch}^{p n+2} \tau-4 a p^{2} n^{2} A^{2 n} B^{2} \operatorname{csch}^{2 p n} \tau \\
& -2 a p n(2 p n+1) A^{2 n} B^{2} \operatorname{csch}^{2 p n+2} \tau \\
& +b v A^{n} B^{4} p n(p n+1)\left\{p^{2} n^{2}+(p n+2)^{2}\right\} \operatorname{csch}^{p n+2} \tau \\
& +b v A^{n} B^{4} p n(p n+1)(p n+2)(p n+3) \operatorname{csch}^{p n+4} \tau \\
& +c_{2} A^{2 n} \operatorname{csch}^{2 p n} \tau=0 .
\end{aligned}
$$

From (27), equating the exponent pairs $(2 n p+2, p n+4)$ or $(2 n p, p n+2)$ give

$$
p=\frac{2}{n}
$$

Equating the exponents of $\operatorname{csch}^{p l} \tau$ and $\operatorname{csch}^{p n} \tau$ yields the requirement that $l=n$. Then, setting the coefficients of $\operatorname{csch}^{p+j} \tau(j=0,2,4)$ to zero, as these are linearly independent functions, gives

$$
\begin{aligned}
4 B^{2}\left(k^{2}-v-4 b v B^{2}\right)-c_{1} & =0, \\
6 B^{2}\left(k^{2}-v-20 b v B^{2}\right)-A^{n}\left(c_{2}-16 a B^{2}\right) & =0, \\
a A^{n}-6 b v B^{2} & =0 .
\end{aligned}
$$

Solving this system yields

$$
\begin{aligned}
A & =\left(\frac{3 c_{1}}{2 c_{2}}\right)^{\frac{1}{n}} \\
B & =\frac{1}{2} \sqrt{\frac{c_{1}\left(b c_{2}+a\right)}{b\left(k^{2} c_{2}-a c_{1}\right)}}, \\
v & =\frac{a\left(k^{2} c_{2}-a c_{1}\right)}{c_{2}\left(b c_{2}+a\right)} .
\end{aligned}
$$

Equation (31) shows that solitons will exist if $c_{1} c_{2}>0$. Also, from (32), the following restriction is obtained

$$
b c_{1}\left(b c_{2}+a\right)\left(k^{2} c_{2}-a c_{1}\right)>0 \text {. }
$$

This, in turn, gives the singular soliton solution which is valid for any positive integer $n$

$$
\begin{aligned}
& u(x, t)=\left(\frac{3 c_{1}}{2 c_{2}}\right)^{\frac{1}{n}} \times \\
& \operatorname{csch}^{\frac{2}{n}}\left[\frac{1}{2} \sqrt{\frac{c_{1}\left(b c_{2}+a\right)}{b\left(k^{2} c_{2}-a c_{1}\right)}}\left(x-\frac{a\left(k^{2} c_{2}-a c_{1}\right)}{c_{2}\left(b c_{2}+a\right)} t\right)\right] .
\end{aligned}
$$

\section{Conclusion}

In this paper, exact soliton solutions to the gO-BBM equation are derived. There are three types of soliton 
solutions - solitary waves, shock waves and singular solitons. These results will be immensely useful in the area of geophysical fluids and/or rotating fluids within the shallow water wave approximation.

These results may be extended in many different ways. For example, the conservation laws can be be studied that will yield the corresponding conserved densities from which conserved quantities can be obtained. Further, perturbation terms will be added to study the dynamics of the gO-BBM equation in the adiabatic approximation.

\section{Acknowledgement}

This work was completed while AB was a Senior Visiting Fellow at UNSW Canberra during April-June 2013. AB is grateful for the hospitality extended to him by the School of Physical, Environmental and Mathematical Sciences.

\section{References}

[1] B. Zhang and Z. Juan, Modeling User Equilibrium and the Day-to-day Traffic Evolution based on Cumulative Prospect Theory, Information Science Letters, 2, 9-12 (2013).

[2] A. Biswas, Quantum Physics Letters, 1, 79-83 (2012).

[3] M. A. Obregon and Y. A. Stepanyants, Mathematical Modelling and Natural Phenomena, 7, 113-130 (2012).

[4] H. A. Zedan and A. A. Saedi, Applied Mathematics and Information Sciences, 4, 253-260 (2010).

[5] Z. Jovanoski, Journal of Nonlinear Optical Physics \& Materials, 10, 79-111 (2001).

[6] Z. Jovanoski, I. N. Towers, S.J. Garth and R. A. Sammut, Journal of Modern Optics, 52, 707-723 (2005).

[7] Z. Jovanoski, I. N. Towers, N. A. Ansari and R. A. Sammut, Optics Communications, 244, 399-409 (2005).

[8] D. R. Rowland and Z Jovanoski, Physical Review B, 54, 13484-13487 (1996).

[9] A. Biswas and S. Konar, Introduction to non-Kerr law solitons, CRC Press, Boca Raton, FL., (2006).

[10] A. Biswas and D. Milovic, Physics of Atomic Nuclei, 74, 781-783 (2011).

[11] L. Girgis and A. Biswas, Applied Mathematics and Computation, 216, 2226-2231 (2010).

[12] S. Johnson and A. Biswas, Physica Scripta, 84, 015002 (2011).

[13] P. Razborova, H. Triki and A. Biswas, Ocean Engineering, 63, 1-7 (2013).

[14] A. Biswas and E. V. Krishnan, Indian Journal of Physics, 85, 1513-1521 (2011).

[15] S. Levandosky, Analysis and Mathematical Physics, 2, $407-$ 437 (2012).

[16] Y. A. Stepanyants, SIGMA, 4, 073 (2008).

[17] A. M. Wazwaz, Communications in Nonlinear Science and Numerical Simulation, 10, 855-867 (2005).

[18] M. Alquran, Journal of Mathematical and Computational Science, 2, 15-22 (2012).
[19] S. Yang and X. Fan, International Journal of Nonlinear Science, 12, 54-59 (2011).

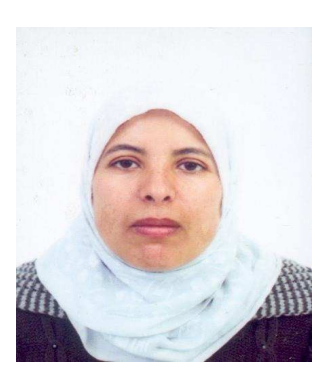

Houria Triki has earned a Ph.D. degree in Physics from Annaba University. Currently, she is a faculty member at Radiation Physics Laboratory at Badji Mokhtar University in Annaba, Algeria. She has published more than 60 papers in various peer-reviewed international journals of high repute and high impact factor. She has also participated in more than 60 scientific meetings.
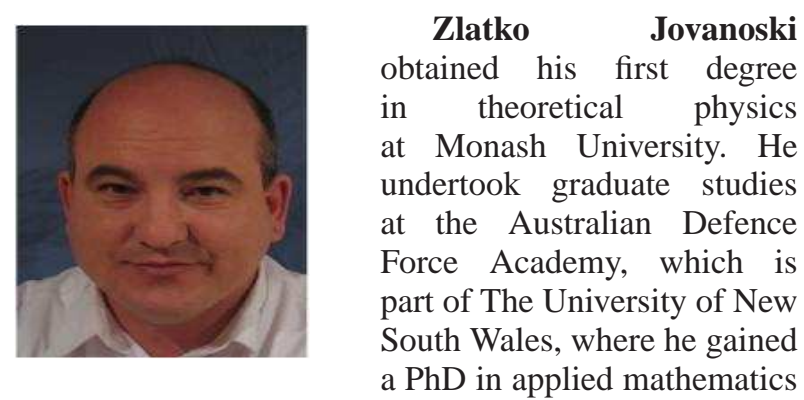
specialising in non-linear optics. His research interests continue to revolve around non-linear optics and the study of non-linear evolution equations. He is currently a Senior Lecture at the University of New South Wales at Canberra.

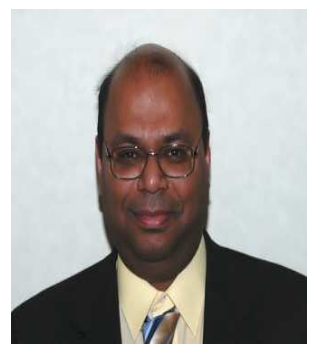

Anjan Biswas earned his B.Sc (honors) degree from Saint Xavier's College in Calcutta, India. Subsequently he received his $\mathrm{M} . \mathrm{Sc}$ and M. Phil degrees in Applied Mathematics from University of Calcutta. Then, he earned MA and Ph.D. degrees in Applied Mathematics from the University of New Mexico in Albuquerque, New Mexico, USA. After that he carried out his Post-doctoral studies in Applied Mathematics from the University of Colorado, Boulder, USA. He was then an Assistant Professor of Mathematics at Tennessee State University that is located in Nashville, TN. Currently; he is working as an Associate Professor of Mathematics at Delaware State University in Dover, DE. His research interest is on Theory of Solitons. 